\title{
Variations
}

Variations

Revue internationale de théorie critique

$24 \mid 2021$

Echos

\section{Theodor W. Adorno sur Marx et les concepts fondamentaux de la théorie sociologique}

\section{Hans Georg Backhaus}

Traducteur : David Buxton, Alexander Neumann et Christophe Magis

URL : https://journals.openedition.org/variations/2060

DOI : 10.4000/variations.2060

ISSN : 1968-3960

Éditeur

Les amis de Variations

Référence électronique

Hans Georg Backhaus, «Theodor W. Adorno sur Marx et les concepts fondamentaux de la théorie sociologique », Variations [En ligne], 24 | 2021, mis en ligne le 03 juillet 2021, consulté le 07 juillet 2021. URL : http://journals.openedition.org/variations/2060 ; DOI : https://doi.org/10.4000/variations. 2060

Ce document a été généré automatiquement le 7 juillet 2021.

Les ami•e•s de Variations 


\title{
Theodor W. Adorno sur Marx et les concepts fondamentaux de la théorie sociologique
}

\author{
Hans Georg Backhaus \\ Traduction : David Buxton, Alexander Neumann et Christophe Magis
}

\section{NOTE DE L'ÉDITEUR}

Le texte suivant est composé des notes prises par Hans Georg Backhaus lors du séminaire d'été d'Adorno en 1962 consacré à Marx. Elles ont été publiées en annexe au livre classique de Backhaus, Dialektik der Wertform : Untersuchungen zur marxschen Ökonomiekritik, Freiburg, Ça ira, 1997 (non traduit en français à l'heure actuelle). La question centrale du rôle de l'abstraction dans l'échange marchand, sera développée plus avant dans une conversation d'Adorno avec Sohn-Rethel sur l'abstraction de l'échange en 1965, traduite dans le présent numéro de Variations.

NB. : Toutes les notes sont des traducteurs.

1 Le «nominalisme social » de [Karl] Popper : Chez Popper, le concept de loi scientifique s'identifie implicitement à la régularité d'occurrences qui se répètent. En vérité, le concept de loi scientifique se rapporte à la codification d'une procédure particulière dans sa structure. Une des caractéristique du positivisme est d'hypostasier la division du travail au sein des sciences, rejetant aussi, ce faisant, le concept de loi ; par exemple quand Popper affirme que l'historiographie ne peut être vérifiée ainsi. Ici, on isole l'historiographie des autres disciplines. On accuse Marx d'économisme. Adorno: Certaines relations intellectuelles s'autonomisent, et si elles sont réduites de manière désinvolte à des causes économiques, on tourne Marx en ridicule. Ce qui compte - c'est notre tâche -, c'est d'expliquer les conditions qui ont mené à l'autonomisation des relations intellectuelles. L'autonomisation intellectuelle elle-même doit être comprise à partir de la dynamique sociale. 
2 Popper reproche à Marx son essentialisme. À cette accusation, Marx aurait ricané, en se réclamant lui-même un nominaliste qui remet Hegel sur ses pieds. Néanmoins, je dirais que Popper a raison sur ce point car, chez Marx, les concepts structurels sont autonomes, sans quoi la diversité sociale ne pourrait être pensée, alors que Popper est essentiellement hostile à la théorie. Dès qu'on renonce au moment de l'autonomie du concept, on nie la possibilité même de la théorie. Celle-ci est alors remplacée par la demande que la sociologie, comprise comme une sorte d'agence sociale, doit fournir des faits bien ordonnés à même d'être utilisés par la praxis dominante.

D'où Popper tire-t-il son exigence d'une «société ouverte »? Après tout, celle-ci se présente comme une notion de sens commun, présentée à la va-vite. Cette notion banale est ici introduite de façon assez naïve et irréfléchie. Tout comme "l'humanité » est déjà un concept général par rapport aux êtres humains individuels.

4 À propos du problème du nominalisme social : L'Aufklärung révèle combien les notions communes sont des fabrications. Les Lumières entendent percer à jour le fauxsemblant d'autonomie de ce que nous fabriquons : c'est l'homme qui produit tout ce qui apparaît comme autonome en lui-même ; il s'agit de thesei et non physei. Popper accuse Marx et Hegel de fétichisme conceptuel suranné, mais il n'existe pas de conscience d'un fait qui n'est pas médié par la conscience.

On refuse de parler dans des termes généraux et on espère alors avoir aboli l'hétéronomie. La représentation sociale est ramenée à de simples faits qui, pour être pensables en tant que faits, doivent être conçus comme étant les produits des individus, tandis qu'en vérité les individus sont regroupés dans des associations qui dépassent l'action concrète de chacun d'entre eux, de telle sorte que ces faits, supposément primaires, sont en réalité eux-mêmes l'objet d'une médiation. Les faits se présentent à nous de manière immédiate, comme s'ils étaient la chose la plus réelle qui soit, alors qu'ils ne sont que des parties d'un tout qui n'est pas donné comme immédiat. Popper ne présenterait pas d'objection à l'étude empirique des institutions. En revanche, si je parlais de l'essence (Wesen), Popper dénoncerait cela comme une mystification théorique. Si je parlais de la structure de notre société comme d'une totalité englobante, alors les positivistes diraient que la société capitaliste n'existe pas, que notre société est pluraliste. Je pose alors la question suivante: est-ce que le sujet qui cherche à accéder à la connaissance ne fait qu'ajouter le concept au matériau existant, ou est-ce que le concept réside à même l'objet que nous observons?

6 Je soulève ici le problème central. Sur ce point, notre approche distingue l'École de Francfort de tous les autres courants de la sociologie. L'échange en lui-même produit l'abstraction. Que les individus s'en rendent compte ou non, dès qu'ils entrent dans un rapport d'échange, par lequel ils rapportent des valeurs d'usage différentes à une valeur- travail, ils actualisent une opération conceptuelle sur le plan social. C'est cela, l'objectivité du concept dans la praxis. Cela démontre que la conceptualité existe non seulement dans l'esprit des philosophes mais qu'elle réside aussi dans la réalité de la chose en soi. De telle sorte que, quand on parle de l'essence (Wesen), on nomme précisément ce que la société contient déjà en elle sans le savoir. Si nous restons assez près des faits, nous finirons par trouver le concept. Il faudra alors revenir au concept présent dans l'objet sans soumettre rétroactivement celui-ci à des concepts classificateurs. C'est lorsque Popper parle de l'aliénation, de l'abstraction, qu'il touche au plus près cet enjeu; alors, selon lui les relations entre êtres humains ont un 
caractère abstrait. Le concept ne doit pas être fétichisé : il est intégré dans un rapport dialectique avec les faits. La structure conceptuelle est elle-même un fait.

7 Les sciences naturelles traitent des objets qui ne possèdent pas de conscience. S'il n'y avait pas de sujets pour réaliser ce type d'abstraction, c'est-à-dire s'il n'y avait pas de sujets pensants, une conceptualité objective ne pourrait pas émerger. Les objets ne se constituent pas en sujets de manière immédiate, mais ils contiennent une dimension subjective qui pousse nécessairement vers l'abstraction. L'objet n'est nullement suffisant en lui-même. On ne devrait pas le poser en principe comme un absolu, parce qu'il ne faut pas oublier qu'il existe une seconde nature qui, nous poussant à nous raidir, nous le rend inaccessible. La domination du social est si forte que la société se présente à nous comme si elle était la véritable nature première. Le positivisme est si aveuglé par la société qu'il confond la seconde nature et la première nature, et met sur le même plan les données sociales et les données des sciences naturelles. À cet égard, notre école s'oppose à tous les courants sociologiques existants.

Quand on dit qu'une part de conceptualité réside dans l'objet, cela ne veut pas dire que la société est fondée sur quelque chose de conceptuel. On ne peut saisir le rapport d'échange sans comprendre la part conceptuelle qu'il contient. Le processus d'abstraction permet de comparer les choses entre elles en faisant apparaître le critère de leur équivalence, sinon l'irrationalité régnerait. La différence entre la société bourgeoise et le féodalisme réside dans le principe de calcul des équivalents. Même si aucun être humain n'avait eu l'idée de cet échange absolu, il existerait toujours objectivement un processus d'abstraction dans l'opération qui consiste à rendre les choses comparables, et qui correspond à l'existence objective du concept d'échange, que les êtres humains y réfléchissent ou non. Le concept s'impose d'autant plus qu'il n'est pas issu de la pensée des individus mais qu'il découle de l'objet lui-même. C'est pour cela que le concept est la chose elle-même, et non la caractéristique particulière subjective subsumée en elle.

Ce type d'objectivité du concept se démarque totalement de l'objectivité enseignée par un réalisme philosophique qui mystifie les concepts, puisque l'objectivité contient en elle tout le nominalisme. La conceptualité contenue dans les rapports d'échange relève elle-même des faits.

Il existe bel et bien une primauté de l'objet sur le concept, de même qu'il existe une primauté de la raison nominaliste sur la conception réaliste. Quand on dit que concept et fait participent tous deux du même processus, cela ne veut pas dire qu'ils ont tous deux la même teneur. L'impénétrable prédomine sur l'autre. Posée ainsi, la pensée ne retombe pas dans une sorte de mythologie.

11 Marx accuse Hegel de transformer le prédicat (c'est-à-dire les opérations, les fonctions) en sujet. Marx se voyait comme un pur nominaliste, mais la structure objective de sa pensée ne l'était en aucun cas.

12 Hegel dit, certes, que le concept d'État précède historiquement le concept de société. Les êtres humains auraient d'abord rencontré la société à travers l'État. Mais le raisonnement de la Philosophie du Droit montre que c'est la dialectique propre de la société qui la fait nécessairement évoluer vers l'État. En d'autres termes, l'État est le produit de la société. 
13 Marx est extrêmement anti-anthropologique, et anti-psychologique. Il s'intéresse plutôt aux institutions qui déshumanisent l'être humain. Il ne développe pas une analyse de l'Homme ; cela aurait été superficiel par rapport à l'être humain historique. La compréhension qu'avait Marx de Hegel est très problématique. Le Marx de la maturité, cependant, a renoué avec l'objectivité du concept, contrairement aux hégéliens de gauche.

15 L'homme est un être vivant qui se reproduit lui-même. L'homme devient homme par lui-même, à travers le travail social. C'est seulement à travers les étapes du travail en société qu'il atteint à son concept, celui d'homme réel, libre.

Marx attribue un concept de l'esprit à Hegel qui est distinct de la sphère matérielle de l'Être. Chez Hegel, l'esprit se décrit comme totalité ; les déterminations du travail ne viennent aucunement d'un principe intellectuel à part. Hegel pense à une confrontation de l'humanité avec la nature, mais interprète le mouvement d'ensemble comme étant celui le mouvement de l'Esprit. Les différentes phases du travail décrivent des aspects matériels, et non pas les activités d'un esprit isolé. Le serviteur (Knecht) n'est pas un intellectuel. Le spirituel réside seulement dans la relation générale qui se révèle entre maître (Herr) et serviteur (Knecht) ${ }^{1}$. L'objectivité (Gegenständlichkeit) a, d'une certaine manière, un sens plus définitif chez Hegel que chez Marx, parce que qu'il conserve une trace ineffaçable de l'institution par rapport à une société libre.

Adorno : (Ici nous touchons au noyau théorique du séminaire) - Que signifie pour Marx la critique de l'économie politique? 1. La critique de la théorie classique du libéralisme, 2. la critique de l'économie en elle-même. Cela amène à la critique de la compréhension qu'a le libéralisme de lui-même (en particulier le livre 4 du Capital, Les Théories sur la plus-value) et à la critique du libéralisme. Marx s'intéresse à une critique immanente de ce dernier. Dans les pays de l'Est, Marx sert à asseoir la domination; ce Marx-là appartient à la littérature de gare. À l'Ouest, on accuse Marx de fonder sa théorie sur l'existence subjective d'une conscience de classe prolétarienne. C'est le contraire de ce que dit Marx. Il confronte la théorie libérale avec sa propre prétention en partant de l'acte d'échange. "Vous dites que des équivalents sont échangés, que l'échange est juste et libre ? Je vous prends au mot, voyons maintenant comment ça marche. » C'est cela, la critique immanente ${ }^{2}$ - car la simple idée que les humains sont transformés en marchandise a déjà été émise par d'autres avant lui. Marx dit : «Il faut faire danser les relations pétrifiées en leur chantant leurs propres mélodies» (Contribution à la critique de la philosophie du Droit de Hegel). Il ne s'agit pas de confronter la société capitaliste avec une autre société, mais de se demander si cette société se conforme à ses propres règles, si elle fonctionne selon les lois qu'elle prétend être les siennes. Or, Marx ne dit pas seulement que celles-ci sont fausses. Il prend au sérieux la dialectique, au lieu de considérer sa terminologie comme simple coquetterie. Dans l'échange, quelque chose apparaît simultanément comme équivalent et comme n'étant pas équivalent. En même temps, l'échange est juste et ne l'est pas. La théorie du libéralisme est conforme à son propre concept, et ce faisant, elle entre en contradiction avec lui. Le rapport d'échange est en réalité pré-conditionné par les rapports de classe : au cœur de la théorie se trouve la présupposition qu'existe une appropriation inégale des moyens de production. Aujourd'hui, cette question n'a presque plus aucune importance dans la discussion de Marx. La critique met à l'épreuve les affirmations en les confrontant à l'objet, et la compréhension de cette situation contradictoire permet ensuite de saisir 
les développements qui en découlent. Le Marx tardif dirait que cette méthode reste encore trop abstraite.

Comme Hegel, Marx voit les stades du développement comme étant qualitativement différents les uns des autres. Rostow ${ }^{3}$, en revanche, ne reconnait pas des structures de base qualitativement différentes. Pour lui, deux stades différents sont vus en termes de plus ou de moins, il n'y a pas de différences qualitatives. Marx n'est pas simplement un historien de l'économie: pour lui, les moments historiques et systémiques sont médiatisés, et le processus historique lui-même est vu comme la transition logique, nécessaire d'une structure à une autre. Marx se démarque non seulement des doctrines statiques, mais aussi des simples historiens qui ne font que décrire des stades successifs. Le concept est entièrement historicisé. Bien que le processus soit formellement idéaliste, il est le déploiement du concept par son propre mouvement: dans le cas de Marx, ce sont les modes de production. Il s'agit d'un double rejet, à la fois de l'idéalisme anhistorique et du positivisme descriptif.

Ce qui caractérise la marchandise, c'est sa valeur d'échange. Ce n'est justement pas le besoin qui est constitutif de la marchandise. La valeur marchande n'est pas dérivée du besoin, mais des conditions objectives de production, dont le besoin est un élément, mais seulement en dernière instance, c'est-à-dire, médiatisé par la nécessité d'écouler la camelote. La théorie objective se caractérise par le fait qu'elle commence avec des institutions, des rapports réels de pouvoir, des processus d'appropriation, plutôt qu'avec des besoins. Vous dites toujours qu'il faut expliquer l'économie à partir des besoins, mais son mécanisme ne sert pas à satisfaire des besoins. Ceux-ci sont plutôt satisfaits au prix d'un grand sacrifice et d'un grincement terrible des engrenages $d u$ système. » Le besoin est seulement entraîné dans ce mouvement : c'est pour cela que l'économie ne doit pas partir des besoins puisque ce monde n'est pas dirigé par eux ; ils ne sont, au contraire, qu'un épiphénomène.

Ce qui est décisif, c'est la primauté de l'appareil de production sur les besoins. Il faut défendre cet argument contre l'objection selon laquelle les phénomènes décrits par Marx pourraient être représentés subjectivement.

21 La méthode de Marx s'attache à compenser des abstractions conceptuelles en les complétant, ensuite, par de vastes différenciations. Voici le problème : est-ce que cela est réconciliable avec la dialectique ou Marx viole-t-il les principes de celle-ci ?

Ce qui rend la marchandise échangeable, c'est l'unité de temps de travail abstrait, socialement nécessaire. Travail «abstrait », parce que le processus qui crée l'unité des valeurs d'usage dans l'équivalence produit une abstraction par rapport aux besoins sociaux.

23 Pour faire ses calculs, l'homme d'affaires ne peut se baser ni sur les conditions dans lesquelles la marchandise a été produite, ni sur ce à quoi elle peut servir; il ne peut se focaliser que sur le temps de travail, le profit, les matériaux. C'est de cela que se compose la marchandise et qui fait d'elle quelque chose de quelque chose de solide, de réel. Le temps de travail abstrait a pour effet, justement, de rendre abstrait les concurrents vivants. En apparence, cette abstraction fait de ce qui s'échange une chose en elle-même. Ce qui est un rapport social apparaît comme la somme des qualités réelles de l'objet. Le concept du fétichisme de la marchandise n'est rien d'autre que le procédé nécessaire d'abstraction. À travers l'abstraction, la marchandise n'apparaît plus comme un rapport social, mais comme si elle avait une valeur en elle-même. 
24 L'échange est toujours la clé de compréhension de la société. C'est une caractéristique de l'économie marchande que ce qui caractérise l'échange - à savoir un rapport entre êtres humains - est rendu invisible et se présente comme s'il s'agissait d'une qualité propre des choses qui s'échangent. Ce n'est pas l'échange qui est fétichisé, mais la marchandise. Ce qui est un rapport social réifié au sein de la marchandise est perçu comme s'il s'agissait d'une qualité naturelle, d'une chose en soi. L'échange n'est pas qu'apparence : il a réellement lieu. L'apparence, dans le processus d'échange, réside dans le concept de survaleur (Mehrwert).

Cela dit, les perceptions fétichisées ne sont pas non plus de simples apparences car, dans la mesure où les humains deviennent dépendants de ces objectivations opaques, la réification n'est pas seulement fausse conscience, mais en même temps réalité, en ce que les humains sont réellement aliénés par la marchandise. Nous sommes réellement dépendants du monde des marchandises. D'une part, le fétichisme de la marchandise est apparence; de l'autre, il est la réalité ultime. La domination de la marchandise réifiée sur l'humanité en est témoin. Que des catégories de l'apparence soient en vérité aussi des catégories de réalité, c'est ça la dialectique.

On ne peut comprendre un concept comme celui de caractère fétiche de la marchandise qu'à la condition de ne pas le limiter à des catégories subjectives. Ici, je ne parle pas de l'attrait qu'exercent sur les individus d'aujourd'hui les marchandises dans un grand magasin. Il ne s'agit pas de la fétichisation psychologique des marchandises singulières, mais de la structure objective de l'économie marchande. Dans une société où la valeur d'échange est le principe dominant, une telle fétichisation se réalise nécessairement. Ce qui est essentiel, c'est que la marchandise disparait en tant que rapport social tandis que toutes les autres réactions de la conscience réifiée s'avèrent secondaires.

Certainement, la fétichisation de la marchandise est la forme de base (Urform) de l'idéologie, mais celle-ci n'est pas simplement la fausse conscience, elle résulte de la structure de l'économie politique. C'est la raison pour laquelle la conscience est déterminée par l'être. Ce qui est décisif, c'est que la structure objective de la forme économique engendre elle-même la fétichisation. C'est cela, le processus objectif de l'idéologie, indépendant de la conscience des individus et de leur volonté. Le sérieux de la doctrine des idéologies (Ideologielehre) ${ }^{4}$ tient au fait que la fausse conscience ellemême apparait comme la forme nécessaire du processus objectif qui maintient la société. La socialisation se réalise à travers cette idéologie. Sur ce point, la question de l'idéologie devient très importante. Et même si nous sommes en mesure de percer la fausse apparence de la société, cela ne modifie pas le caractère fétichiste de la marchandise : tout homme d'affaires qui fait ses calculs est obligé de se comporter d'une manière conforme à ce fétiche. Sinon, il coule !

L'argent n'est qu'un symbole de travail ossifié et non une chose en soi : ainsi les processus de la finance ne sont-ils pas primaires, il faut plutôt les déduire de l'économie politique. Quand la valeur d'échange s'autonomise, on peut la viser comme une chose en soi. Le sens de la formule A-M-A' est cette réification de la valeur d'échange ${ }^{5}$.

29 La question cruciale est d'où vient la survaleur (Mehrwert) ? La sphère de la circulation est secondaire, car la survaleur y existe déjà. Dans la sphère de la circulation, les entrepreneurs se bousculent pour la survaleur qui est, cependant, déjà produite. 
30 La force de travail est la source de la survaleur, car la force de travail possède à la fois une valeur d'usage et d'échange. C'est cela, le noyau de l'affaire. Le travailleur est libre dans la mesure où il peut bouger d'un secteur à un autre.

31 La valeur elle-même se définit comme étant du travail socialisé. Et c'est bien pour cela que les machines ne peuvent produire d'elles-mêmes de la valeur. Ce que les machines produisent renvoie au travail, car elles sont elles-mêmes produites par le travail. Les entrepreneurs courent après la survaleur absolue, mais non parce qu'ils sont méchants. L'explication psychologisante est aussi étrangère à Marx qu'à Hegel.

32 La théorie de Marx du « masque de caractère » (Charaktermaske) 6 pose le concept de rôle social (Rollenbegriff), mais celui-ci dérive des conditions objectives : le rôle est imposé au sujet par la structure. De nos jours - chez Parsons par exemple ${ }^{7}$ - il n'y a pas de réflexion sur le concept de rôle, mais plutôt une absolutisation de celui-ci. La raison pour laquelle je suis sceptique à propos du concept de rôle est qu'il n'est pas compris comme un moment nécessaire dans un processus, mais plutôt isolé, singularisé.

Voici le noyau de la dialectique: Les capitalistes sont forcés d'accumuler de la survaleur, par tous les moyens. À cette fin, ils sont poussés à développer des machines afin de remplacer le travail vivant par le travail mort. S'ils ne le font pas, ils périront dans la lutte concurrentielle. Ici, un aspect de la sphère de la circulation agit rétroactivement sur la sphère de la production. Puisque les capitalistes sont contraints, ils créent ainsi les conditions par lesquelles les forces productives pourront s'affranchir des chaînes de l'économie capitaliste. Ensuite, ce faisant, ils mettent en branle une dynamique qui se retourne contre eux : de plus en plus, une quantité de travail devient surnuméraire, participant à la formation des crises et menaçant sans cesse le système lui-même. Afin de se maintenir, le système doit produire précisément de telles tendances par lesquelles il sape sa propre existence. Le rôle de la spontanéité consiste à maitriser ce processus dialectique aveugle qui, mène à la destruction du système dans sa totalité, afin d'élever le tout vers un mode de production supérieur qui dépasse (aufheben) le précédent. La dialectique, dans la mesure où elle est aveugle, crée alors les conditions qui permettent autre chose. Si nul moment de liberté n'intervient, c'est-àdire, si le mouvement d'ensemble est abandonné à lui-même, il dépérit ${ }^{8}$.

L'insécurité permanente est l'une des raisons du désir rétrograde en faveur de modes d'existence agraires ou artisanaux. C'en est la signification authentique. L'autre signification, mystifiante, est fausse parce que ces rapports ne peuvent être rétablis.

35 Afin de comprendre le concept de survaleur, il faut comparer deux temporalités : le temps nécessaire à la production de la force de travail et le temps pendant lequel le travailleur fournit effectivement son travail. Il ne faut pas partir de la marchandise produite par le travailleur car il s'agit plutôt d'un processus d'échange : le travailleur vend son temps de travail pour lequel il reçoit un équivalent. Mais le temps qu'il donne, et le temps nécessaire pour la reproduction de sa force de travail sont différents. D'une part, l'échange a lieu sous la forme d'équivalents: le travailleur donne son temps de travail et reçoit en retour ce qu'il faut pour la reproduction de sa force de travail. C'est là que se trouve la source de la survaleur, sans que l'on doive prendre en considération la nature de la marchandise produite. On échange des choses équivalentes et, en même temps, des choses qui ne le sont pas. Derrière cela se trouve la totalité des rapports de classe. Le travailleur accepte ces conditions seulement parce qu'il ne possède rien d'autre que sa force de travail. Derrière cet échange insolite se trouve la question des rapports de classe. 

d'expliquer le mécanisme entier de l'économie en termes de besoin ${ }^{9}$. Une telle explication subjective peut se faire si l'on se contente d'une esquisse formaliste des processus économiques. Mais dans ce contexte, il ne faudrait pas faire abstraction de la puissance sociale ou de l'impuissance. Le contrôle de la consommation n'est pas une invention récente, même si de nouvelles formes de régulation prévalent aujourd'hui. Mais la consommation n'est pas la clé pour la compréhension de l'économie, parce que les possibilités de consommation des individus dépendent du système économique global (on ne peut consommer que ce que permet son statut social), et de la situation générale de l'économie. consommation) représente d'une façon limpide le circuit économique, mais de savoir quelle théorie exprime correctement la réalité dans laquelle les relations économiques des hommes s'inscrivent. Une approche qui ne prend pas en compte la dépendance du consommateur au système global n'est pas adéquate à la réalité. On peut démontrer que les changements dans les habitudes de consommation ne viennent pas du sujet, mais sont des processus objectifs qui ont leurs racines dans la structure sociale. C'est pourquoi Marx part, non de la consommation, mais de la production, comprise comme la domination des propriétaires des moyens de production. Cette approche correspond davantage à la réalité.

38 Choisir une approche plutôt qu'une autre n'est jamais neutre. Le meilleur système théorique est celui qui peut intégrer le plus de rapports réels en son sein. Si ces rapports sont antagonistes (système des classes), il faut alors que ces antagonismes puissent s'exprimer dans la théorie.

39 La science économique subjective est essentiellement une analyse des activités marchandes qui présuppose l'existence d'un marché. Engels invoque avec justesse l'héritage de la philosophie allemande : la question qu'il posait alors était de savoir ce qui constitue la survaleur, en partant des conditions immanentes qui ont permis l'émergence du système, tandis que la doctrine subjective se contente de décrire de manière élégante tout ce qui existe déjà.

40 À l'opposé, Marx ne s'intéresse pas à la description d'une société marchande, mais aux éléments constitutifs de l'expérience sociale, et à la critique des catégories de l'activité économique. Cette approche, plus profonde, qui exprime mieux la réalité, pose le problème de la constitution de la société marchande. L'enjeu en est la possibilité de saisir les éléments constitutifs de la totalité. Cette question de la constitution apparait déjà dans l'arbitraire avec lequel des coupes analytiques sont imposées à la réalité d'une manière abstraite. La doctrine subjective de la science économique est essentiellement une apologie du système. L'analyse de la question du prix est un épiphénomène en regard de la question de la constitution.

41 Passons à la critique de Marx : On ne peut s'arrêter au phénomène de l'aliénation, cette dernière étant en soi une catégorie idéaliste. L'aliénation résulte, cependant, du caractère marchand de l'économie. La question du pouvoir ne peut pas non plus être abordée dans des termes abstraits, elle s'impose plutôt à travers la reproduction de la vie matérielle. S'il s'agissait simplement d'une question d'aliénation et de pouvoir, Marx n'aurait pas grand-chose à nous dire, l'hégélianisme de gauche serait tout ce qui reste de lui. Mais Marx voulait exposer à la critique la manière dont le pouvoir et l'aliénation se présentent dans une société concrète. 


\section{NOTES}

1. Référence au chapitre sur « Domination et servitude » de la Phénoménologie de l'esprit de Hegel.

2. Sur l'importance de la critique immanente du capitalisme moderne, voir Th. W. Adorno, «Sur la logique des sciences sociales", in De Vienne à Francfort : la querelle allemande des sciences sociales, Bruxelles, Complexe, Bruxelles, 1979.

3. Walt Whitman Rostow (1916-2003), économiste et politiste américain, auteur des Étapes de la croissance économique: un manifeste anti-communiste (1960). Rostow y propose une théorie du développement (capitaliste) en cinq étapes : la société traditionnelle, les conditions préalables au décollage, le décollage, la phase de maturité, l'âge de la consommation de masse. La notion de "pays en voie du développement» en est dérivée. Favorable à l'intervention américaine au Vietnam, il fut conseiller pour la Sécurité nationale, 1966-69.

4. Nous reprenons ici la proposition de traduction faite dans le premier recueil en français des écrits sociologiques d'Adorno, Société : Intégration - désintégration, Paris, Payot, 2011 pour le texte «Beitag zur Ideologielehre » : «Contribution à la doctrine des idéologies ».

5. Référence au schéma marxien de la circulation dans le mode de production capitaliste : à la différence des modes de production précédents, où l'argent servait de médiation entre différentes marchandises ( $\mathrm{M}-\mathrm{A}-\mathrm{M}$ - c.-à.- $\mathrm{d}$ que les individus avaient produit une marchandise et passaient par la médiation de l'argent pour la vendre afin de s'en acheter une autre), dans le mode de production capitaliste, la circulation se caractérise par le fait que c'est la marchandise qui y devient la médiation entre l'argent et plus d'argent (A-M-A' - c.-à.-d. que le capitaliste achète n'importe quelle marchandise pour la revendre, plus cher). 
6. Ce terme de Marx désigne les masques sociaux que revêtent les gens au-delà des rapports de production (voir Le Capital, PUF, 1993, p. 89). Adorno, pour sa part, s'oppose au concept non critique de "rôle », "prélevé, sans analyse, de la façade sociale » (Société: Intégration, Désintégration, Paris, Payot, 2011, p. 27-28).

7. Talcott Parsons (1902-79), sociologue américain, fondateur de l'école structuro-fonctionnaliste, à la fois empirique et systématique, qui a dominé la sociologie américaine jusqu'aux années 1960 . Traducteur de Max Weber. Contre le déterminisme social, il a développé une théorie de l'action selon laquelle les sujets s'orientent en fonction des valeurs qu'ils se sont eux-mêmes choisies, l'ensemble social étant défini comme un ensemble d'actions.

8. La notion de spontanéité chez Adorno s'oppose à la normalisation et à la standardisation, notamment de l'industrie culturelle, et permet de sortir de l'identité conceptualisée entre sujet et objet. Voir l'article de Christophe Magis dans le présent numéro.

9. Dans ce séminaire, la «théorie subjective » réfère à la science économique « bourgeoise », qui ne dépasse pas les apparences. Sur la distinction opérée par Adorno entre «théorie objective » et " théorie subjective » dans les sciences sociales, voir « Intervention lors de la discussion à propos de "Capitalisme tardif ou société industrielle" ", in Société, op. cit., p. 109-21:

«...[C]e qui est visé ici par une sociologie objective, c'est une sociologie qui croit pouvoir recourir à des structures de la société prélevées sur le système de la société lui-même ou touchant au système de la société lui-même - et non aux structures qui sont des systématisations ou des schémas d'ordonnancement générés par le besoin ou l'organisation scientistes. Face à cela, ce sont deux sortes de choses que j'ai désignées par le terme subjectif.

Tout d'abord, le subjectivisme scientiste, où les catégories d'ordonnancement propres à la science classificatoire sont censées fournir les véritables vecteurs de la connaissance, au détriment de la chose même. Deuxièmement [...] le terme désigne l'activité qui consiste à recourir à des données subjectives - donc par exemple à des tranches de revenu ou à la conscience du statut social, à la conscience du rôle, voire aux indices bien connus utilisés par la sociologie américaine des stratifications -, alors qu'en vérité, il s'agit de positions objectivement prédéfinies que des hommes singuliers occupent au sein du processus de production. » (p. 112).

10. Helmut Schelsky (1912-94), sociologue allemand conservateur, ancien nazi, défenseur d'une approche empirique et descriptive, contestait qu'une société divisée en classes sociales existait toujours.

La référence à la paupérisation relative renvoie au débat historique (1896-1903) entre, d'un côté, Édouard Bernstein, l'un des dirigeants du parti social-démocrate allemand (à l'époque d'obédience marxiste), qui a appuyé son appel au réformisme sur le constat que le pronostic marxiste de la paupérisation absolue du prolétariat s'est avéré faux, et de l'autre, le théoricien Karl Kautsky, qui a avancé la thèse de la paupérisation relative : même si le niveau de vie des prolétaires s'améliore, l'inégalité entre la bourgeoisie et le prolétariat s'accroit. Le congrès des sociaux-démocrates de 1903 a tranché en faveur de Kautsky. Adorno renvoie dos à dos les deux positions; pour lui, il s'agit d'un concept strictement économique, qui ignore des facteurs politiques comme des allocations consenties, et des facteurs externes comme les profits coloniaux. Voir « Réflexions sur la théorie des classes » (1942, non publié du vivant d'Adorno) in Société, op. cit., p. 69-72, et la note 34 des traducteurs, p. 81-2. 